\title{
Control Metabólico en Pacientes Diabéticos Tipo 2: grado de Control y nivel de Conocimientos (Estudio Azuer)
}

\author{
Pedro Domínguez Sánchez-Migallón ${ }^{\mathrm{a}}$ en representación del Grupo AZUER ${ }^{\mathrm{b}}$
}

\begin{abstract}
a Médico de Familia. Centro de Salud Manzanares 2, Manzanares (Ciudad Real, España).

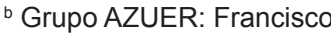
Alhambra García Cervigón (Médico), José Camacho Sánchez-Migallón (DUE), Pedro Domínguez Sánchez-Migallón (Médico), Antonio Fernández Simancas (DUE), Rafael V. García García (Médico), Raimundo Garrido Garrancho (DUE), Josefa López-Peláez Borja (DUE), Julia Maeso González DUE), Rafael Medina de la Casa (Médico), Ana Morillo Castro (Médico), Salvador Pérez Martín (DUE), Ana María Román Ruiz del Moral (Médico), María Sanabria Moreno (DUE), Ignacio M. Sánchez Barrancos (Médico), Francisca Vázquez Gallego (DUE), Domingo Zamora Martín (Médico)
\end{abstract}

Correspondencia: Pedro Domínguez Sánchez-Migallón, Centro de Salud Manzanares 2, Carretera de La Solana, s/n 13200 - Manzanares (Ciudad Real). Correo electrónico: pdsanchez@sescam.jccm.es.

Recibido el 7 de noviembre de 2010.

Aceptado para su publicación el 14 de enero de 2011.

\section{RESUMEN}

Objetivo. Conocer el grado de control y nivel de conocimiento del control metabólico (ADA 2009) de los diabéticos tipo 2 (y de aquellos que son miembros de asociación de pacientes) y si un mayor conocimiento se relaciona con un mejor control metabólico.

Material y métodos. Estudio observacional, descriptivo y transversal en Atención Primaria (Centro de Salud Manzanares II) sobre 297 diabéticos (138 hombres y 159 mujeres), mediante recogida de datos de historia clínica (sociodemográficos, clínicos, analíticos y de repercusión) y aplicación de un cuestionario sobre conocimiento de objetivos de control, relacionando el conocimiento con el grado de control.

Resultados. La prevalencia de diabetes mellitus es de 8,48\% (IC 95\%: 8,03-8,94]. El 98\% de la mujeres son "sin estudios" ( $p<0,0001)$ o con "estudios primarios" $(p=0,01)$. El 18,5\% (IC 95\%: $14,3-24,5)$, y además más jóvenes, pertenecen a asociaciones de diabéticos. El tabaquismo y la arteriopatía periférica son más frecuentes en varones. Aproximadamente el $50 \%$ tienen controlada la glucemia y la $\mathrm{HbA}_{1} \mathrm{C}$, y el $42 \%$ la PAS. Sólo el $7,07 \%$ cumplen todos los objetivos. Conocen los objetivos (relacionado con nivel educativo: $p<0,001$ ) de glucemia el $48,7 \%$, de glucemia postprandial el $16,5 \%$ y de $\mathrm{HbA}_{1} \mathrm{C}$ el $7 \%$, siendo más elevado (32,7\% y $29 \%$ respectivamente) en los pertenecientes a asociaciones. La antiagregración, el bloqueo del SRA-A y las estatinas son empleados en un $45,8 \%, 65,3 \%$ y $44,8 \%$ respectivamente.

Conclusiones. El control metabólico de los diabéticos es mejorable. El conocimiento por los pacientes de los objetivos de control está relacionado con el nivel educativo, pero es muy bajo, e incluso mejorable en los miembros de asociaciones. Es necesario formar a los pacientes en estos aspectos, lo que posiblemente influya en un mejor control metabólico.

Palabras clave. Diabetes Mellitus, Control, Conocimiento.

\section{ABSTRACT}

Metabolic Control in Patients with Type 2 Diabetes: degree of Control and level of Knowledge (Azuer Study).

Objective: To determine the degree of metabolic control and the level of knowledge of such control (ADA 2009) in type 2 diabetic patients (and those who are members of patient associations) and if a greater knowledge correlates with improved metabolic control.

Material and methods. Cross sectional, observational, descriptive study in Primary care (Manzanares II Health Centre) in 297 diabetics (138 men and 159 women), using data from their medical records (sociodemographics, clinical, analytical and repercussions) and a questionnaire on the knowledge of metabolic control objectives, correlating knowledge with degree of control. Results. The prevalence of diabetes mellitus type 2 is $8.48 \%(95 \% \mathrm{Cl}: 8.034-8.94) ; 98 \%$ of the women have "no education" $(p<0.0001)$ or only "primary education" $(p=0.01)$ and $18.5 \%$ (95\% Cl: $14.3-24.5)$ belong to diabetic associations and tend to be younger patients. Smoking and peripheral arteriopathy are more frequent in men. Blood sugar and $\mathrm{HbA}_{1} \mathrm{C}$ are controlled in approximately $50 \%$ and SBP in $42 \%$. Only $7.07 \%$ knew all the metabolic control objectives; $48.7 \%$ knew the blood sugar control objectives (related with educational level: $p<0.001$ ), $18.5 \%$ those of postprandial blood sugar and $7 \%$ of $\mathrm{HbA}_{1} \mathrm{C}$ levels. This percentage being higher in those belonging to associations $(32.7 \%$ and $29 \%$, respectively). Anti-platelets, RAAS inhibitors and statins were used in $45.8 \%, 65.3 \%$ and $44.8 \%$ of patients, respectively.

Conclusions. Metabolic control of diabetic patients is improvable. Patient's level of knowledge of metabolic control is related with educational level, but it is very low, and can be improved even in those belonging to associations. Patients need to be educated in metabolic control which would probably result in an improvement of such control.

Key words. Diabetes Mellitus, Control, Knowledge. 


\section{INTRODUCCIÓN}

La diabetes mellitus (DM) es una alteración metabólica caracterizada por una elevación de la glucemia y por complicaciones microvasculares y cardiovasculares que incrementan sustancialmente la morbimortalidad asociada con la enfermedad y reducen la calidad de vida (ADA 2000). La prevalencia de este trastorno se incrementa ${ }^{1}$ paulatinamente debido a cambios de hábitos (alimenticios y de estilo de vida), cambios en los criterios diagnósticos, envejecimiento de la población, menor mortalidad de los pacientes diabéticos y, finalmente, un verdadero aumento de la incidencia.

La DM se suele asociar a otros factores de riesgo cardiovascular (FRCV), y el riesgo global de un paciente diabético es considerado equivalente al de aquél con cardiopatía isquémica ${ }^{2}$. El adecuado control metabólico en nuestros diabéticos disminuye la incidencia de las complicaciones micro y macrovasculares (estudio UKPDS ${ }^{3}$ ), pero el control metabólico obtenido en ellos dista mucho de ser óptimo, pues según diversos estudios suele ser deficiente, aunque variable, y se detecta una infrautilización de medicación para el control de los FRCV asociados $^{4-7,10}$. El estudio ELIPSE ${ }^{8}$, realizado en nuestra provincia, concluyó que el grado de control de los FRCV en diabéticos tipo 2 en Atención Primaria (AP) no garantiza una prevención cardiovascular correcta, y aconsejó un uso más intensivo del tratamiento farmacológico.

Los criterios de control metabólico adecuado en pacientes diabéticos según las sociedades científicas como la $\mathrm{ADA}^{9}$ son: glucemia basal $<110 \mathrm{mg} /$ $\mathrm{dl}$, glucemia postprandial $130-180 \mathrm{mg} / \mathrm{dl}$, hemoglobina glicada menor de $7 \%$, presión arterial sistóli$\mathrm{ca} /$ diastólica $<130 /<80$, colesterol total $<185 \mathrm{mg} / \mathrm{dl}$, HDL-colesterol $>40 \mathrm{mg} / \mathrm{dl}$, LDL-colesterol $<100 \mathrm{mg} /$ dl, triglicéridos $<150 \mathrm{mg} / \mathrm{dl}$, no fumar y realizar ejercicio físico de tipo aeróbico al menos 150 minutos/ semana.

Existe consenso en que el control del paciente diabético requiere usualmente varios fármacos para el control de los FRCV: antagonistas del sistema renina-angiotensina (SRA-A), antiagregantes ${ }^{9}$ en muchos casos y estatinas ${ }^{4}$. También sabemos que el desconocimiento de la propia enfermedad influye desfavorablemente en el cumplimiento terapéutico y éste a su vez en el grado de control ${ }^{11}$.

La intervención educativa mejora los niveles de hemoglobina glicosilada, al menos inicialmente ${ }^{12}$.
El mayor nivel de conocimientos se observa en pacientes de menor edad, mayor nivel académico y menos de 10 años de evolución de la enfermedad ${ }^{13}$. Si bien los diabéticos suelen tener un bajo nivel de conocimientos sobre su enfermedad ${ }^{14}$, la intervención de educación diabetológica proporciona mejoría en el control glucémico en ayunas ${ }^{15}$, y en el nivel de conocimientos ${ }^{16}$, aunque estudios realizados en nuestro país no muestren mejoría del control metabólico ni prevención de complicaciones. Otros, en cambio $^{17}$, sí hallaron mejoría en el control metabólico (en pacientes con mal control previo) e incremento en el nivel de conocimientos.

El objetivo principal de nuestro estudio es conocer el grado de control de los pacientes diabéticos tipo 2 de nuestra zona básica de salud, siendo objetivos secundarios valorar el nivel de conocimiento sobre el control metabólico que tienen ellos, establecer la relación entre el nivel de conocimiento y el grado de control metabólico y comprobar si aquellos pacientes que pertenecen a asociación de diabéticos conocen mejor los objetivos de control de su enfermedad.

\section{MATERIAL Y MÉTODO}

Se trata de un estudio observacional, descriptivo y transversal en el ámbito de AP (Zona de Salud Manzanares II, que comprende la población de Membrilla y más del $50 \%$ de la población de Manzanares), siendo el periodo de estudio del 1 Septiembre de 2009 al 30 de Abril de 2010.

La población analizada fueron los diabéticos tipo 2 de la Zona de Salud identificados en las Historias Clínicas del Programa Turriano usado en AP en el Servicio de Salud de Castilla-La Mancha (SESCAM). Se excluyeron los pacientes diabéticos tipo 1 , desplazados o adscritos temporalmente al cupo médico, fallecidos (aunque figurasen en la base de datos), no respondedores a las preguntas planteadas, así como aquellos que por su avanzada edad y/o estado cognitivo no pudieron contestar. Para el cálculo del tamaño muestral se empleó la fórmula de proporciones en poblaciones finitas (donde $p=0,25$ y $q=0,75$, con una confianza del $95 \%$ y un error muestral del $5 \%$, obteniéndose un $\mathrm{N}=288$, que incrementado en un $15 \%$ ante posibles pérdidas supuso una muestra final de 331 pacientes.

La selección de éstos se realizó mediante muestreo sistemático sobre el listado de diabéticos de cada uno de los cupos médicos obtenidos de la base de 
datos de Turriano. De cada paciente seleccionado se rellenó una Hoja de Recogida de Datos (HRD) diseñada ad hoc, donde se registraron datos sociodemográficos (sexo, edad, antigüedad del diagnóstico, nivel de escolaridad), pertenencia o no a asociación de diabéticos, realización de automedida de la glucosa sanguínea (AMGS), FRCV asociados, repercusión macro y microvascular de la DM, valores analíticos y antropométricos (considerados como válidos si tenían una antigüedad igual o infe-

\begin{tabular}{|c|c|c|c|}
\hline Parámetro & Hombres & Mujeres & Significación \\
\hline $\begin{array}{l}\text { Sexo } \\
\text { Edad (DE) } \\
\geq 65 \text { años } \\
\text { Diagnóstico hace (años) (DE) } \\
\text { Nivel de escolaridad } \\
\text { - Sin estudios (29,2\%) } \\
\text { - Primarios (60,9\%) } \\
\text { - Medios }(8,1 \%) \\
\text { - Universitarios (1,8\%) } \\
\text { Miembro asociación (SI) } \\
\text { Auto Medición Glucosa (SI) } \\
\text { Antecedentes Personales } \\
\text { - Hipertensión Arterial } \\
\text { - Dislipemia } \\
\text { - Obesidad } \\
\text { - Tabaquismo } \\
\text { Repercusión DM } \\
\text { - Cardiopatía Isquémica } \\
\text { - Arteriopatía Periférica } \\
\text { - Enf. Cerebro-Vascular } \\
\text { - Retinopatía } \\
\text { - Nefropatía } \\
\text { - Neuropatía }\end{array}$ & $\begin{array}{c}\mathrm{n}=138 \\
66,6(10,34) \\
61,5 \%(52,9-69,7) \\
7,54(6,54) \\
\\
12,4 \%(7,3-18,9) \\
68,5 \%(60,4-76,4) \\
15,3 \%(9,6-22,3) \\
3,6 \%(1,2-8,2) \\
16,2 \%(10,2-23,1) \\
47,0 \%(38,5-55,7) \\
\\
69.6 \%(61,1-77,1) \\
51.5 \%(42,8-60) \\
55,1 \%(46,4-63,5) \\
22.5 \%(15,8-30,3) \\
\\
13,8 \%(8,5-20,6) \\
11,6 \%(6,6-18,1) \\
7,2 \%(3,5-12,9) \\
5,1 \%(2,0-10,1) \\
9,4 \%(5,1-15,5) \\
9,4 \%(5,1-15,5)\end{array}$ & $\begin{array}{c}\mathrm{n}=159 \\
71,9(11,1) \\
76,7 \%(69,3-83,6) \\
9,34(6,85) \\
\\
43,9 \%(36,1-52,1) \\
54,1 \%(46,1-62,0) \\
1,9 \%(0,3-5,4) \\
0 \\
20,7 \%(14,7-27,8) \\
45,8 \%(37,9-53,9) \\
\\
77.3 \%(70,0-83,6) \\
60,3 \%(52,3-68,0) \\
59,7 \%(51,6-67,0) \\
6,3 \%(3,0-11,2) \\
\\
11,3 \%(6,8-17,3) \\
5,0 \%(2,2-9,7) \\
11,3 \%(6,8-17,3) \\
3,8 \%(1,4-8,0) \\
18,2 \%(12,5-25,1) \\
8,2 \%(4,4-13,5)\end{array}$ & $\begin{array}{c}<0,0001 \\
0,0045 \\
\text { NS } \\
<0,0001 \\
0,01 \\
<0,0001 \\
\text { NS } \\
\text { NS } \\
\text { NS } \\
\text { NS } \\
\text { NS } \\
<0,0001 \\
\\
\text { NS } \\
0.0385 \\
\text { NS } \\
\text { NS } \\
0.0296 \\
\text { NS }\end{array}$ \\
\hline
\end{tabular}

Tabla 1. Características de la muestra.

\begin{tabular}{|c|c|c|c|}
\hline Parámetro & Hombres & Mujeres & Significación \\
\hline Glucemia basal & $138.04(38.4)$ & $141.08(39.7)$ & NS \\
\hline $\mathrm{Hb} \mathrm{A} 1 \mathrm{C}$ & $7.17(1.17)$ & $7.26(1.24)$ & NS \\
\hline PA Sistólica & $135.58(15.6)$ & $134.48(17.4)$ & NS \\
\hline PA Diastólica & $77.18(8.8)$ & $75.27(8.05)$ & 0.055 \\
\hline Col Total & $193.07(37.6)$ & $198.27(39.4)$ & NS \\
\hline HDL Col & $45.89(9.7)$ & $49.3(10.9)$ & 0.0059 \\
\hline LDL col & $117.19(33.66)$ & $121.43(30.9)$ & NS \\
\hline Triglicéridos & $148.22(86.0)$ & $146.57(69.7)$ & NS \\
\hline Creatinina & $0.99(0.24)$ & $0.98(0.60)$ & NS \\
\hline MAU & $30.87(151,3)$ & $35.60(270.2)$ & NS \\
\hline Filtrado Glomerular estimado (MDRD) & $79.93(20,2)$ & $68.53(26.14)$ & 0.0001 \\
\hline $\mathrm{IMC} \geq 30$ & $78(56.5 \%)$ & $104(65.4 \%)$ & NS \\
\hline Perímetro abdominal & $109.75(11,10)$ & $103,86(11,8)$ & 0.0001 \\
\hline Realización de ejercicio & $98(74,24 \%)$ & $92(59,74 \%)$ & 0.006 \\
\hline Ejercicio (min./semana) & 337.19 (215.49) & $268.9(153.56)$ & 0.0132 \\
\hline Ejercicio $\geq 150 \mathrm{~min} . / \mathrm{sem}$ & $62.3 \%$ & $47.1 \%$ & 0,008 \\
\hline \multicolumn{4}{|l|}{ Enfermedad Renal Crónica } \\
\hline - Grado $1+M A U=>30 \mathrm{mg}$ & $4,3 \%$ & $0,6 \%$ & 0,03 \\
\hline - Grado $2+\mathrm{MAU}=>30 \mathrm{mg}$ & $6,5 \%$ & $0,6 \%$ & 0,004 \\
\hline - Grado 3 (FGe:30-59 ml/min) & $17,9 \%$ & $35,4 \%$ & 0,007 \\
\hline - Grado 4 (FGe:15-29 ml/min) & 0 & $4,5 \%$ & - \\
\hline - Grado 5 (FGe: < $15 \mathrm{ml} / \mathrm{min}$ ) & 0 & $1,2 \%$ & - \\
\hline - Total (\%) ERC & $28,7 \%(21,6-37,3)$ & $42,1 \%(34,3-50,2)$ & 0,013 \\
\hline
\end{tabular}

Tabla 2. Valores analíticos. 


\begin{tabular}{|c|c|c|c|}
\hline Parámetro & $\begin{array}{c}\text { Hombres } \\
(n=138)\end{array}$ & $\begin{array}{l}\text { Mujeres } \\
(n=159)\end{array}$ & Significación \\
\hline Glucemia ( $\leq 130 \mathrm{mg} / \mathrm{dl})$ & $48,5 \%(39,9-57,2)$ & $43,3 \%(35,5-51,4)$ & NS \\
\hline $\mathrm{HbA} 1 \mathrm{C}(\leq 7 \%)$ & $50 \%(41,3-58,6)$ & $50,9 \%(42,9-58,9)$ & NS \\
\hline P A Sistólica $(\leq 130$ mm Hg) & $42,0 \%(33,6-50,7)$ & $44,0 \%(36,1-52,1)$ & NS \\
\hline P A Diastólica ( $\leq 80 \mathrm{mmHg})$ & $67,4 \%(58,9-75,1)$ & $79,2 \%(72,1-85,2)$ & NS \\
\hline Colesterol Total ( $\leq 185 \mathrm{mg} / \mathrm{dl})$ & $42,0 \%(33,6-50,7)$ & $39,6(31,9-47,6)$ & NS \\
\hline $\mathrm{HDL}-\mathrm{Col}(\mathrm{H}>40 / \mathrm{M}=>50 \mathrm{mg} / \mathrm{dl})$ & $69,5 \%(61,1-77,1)$ & $43,6 \%(35,5-51,4)$ & 0,0002 \\
\hline LDL-Colesterol ( $\leq 100$ mg/dl) & $34,0 \%(26,2-42,0)$ & $25,1 \%(18,6-32,6)$ & NS \\
\hline Triglicéridos (s150 mg/dl) & $63,0 \%(54,4-71,1)$ & $61,0 \%(52,9-68,6)$ & NS \\
\hline Tabaquismo(No fumador/a) & $77,5 \%(69,6-84,2)$ & $93,7 \%(88,7-96,9)$ & $<0,0001$ \\
\hline \multicolumn{4}{|l|}{ Parámetros controlados * } \\
\hline - (A) Glucemia basal & $48,5 \% \quad(39,9-57)$ & $43,3 \% \quad(33,5-51,4)$ & NS \\
\hline$-(B)=(A)+H_{b A_{1}} C$ & $46,4 \%(37,8-55)$ & $41,5 \%(33,7-49,5)$ & NS \\
\hline$-(C)=B+$ PA Sistólica & $22,4 \%(15,8-30,3)$ & $21,3 \%(15,2-28,5)$ & NS \\
\hline - (D) = C + PA Diastólica & $19,6 \%(13,3-27,1)$ & $18,9 \%(13,1-25,8)$ & NS \\
\hline$-(E)=D+L D L$ colesterol & $12,3 \%(7,3-18,9)$ & $5,0 \%(2,2-9,6)$ & 0,02 \\
\hline$-(F)=E+$ No fumador & $9,4 \%(5,0-15,5)$ & $5,0 \%(2,2-9,6)$ & NS \\
\hline
\end{tabular}

Tabla 3. Proporción de pacientes controlados en los diferentes parámetros (IC 95\%). *American Diabetes Asociation, 2009.

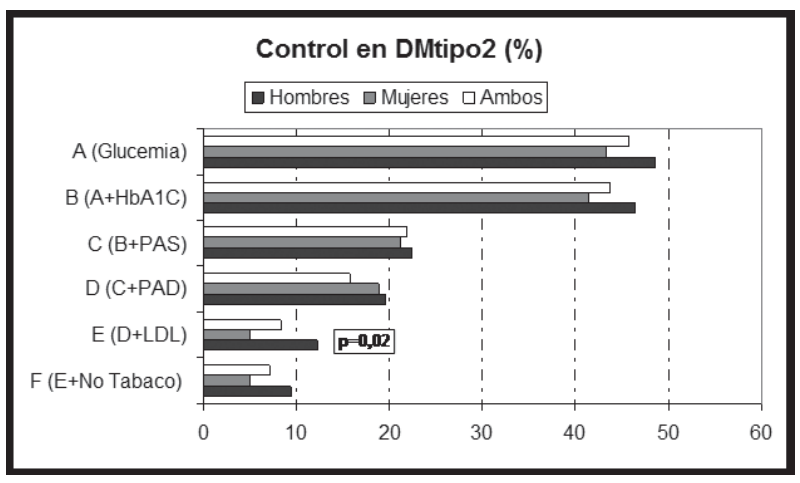

Figura 1. Control acumulativo de parámetros (\%).

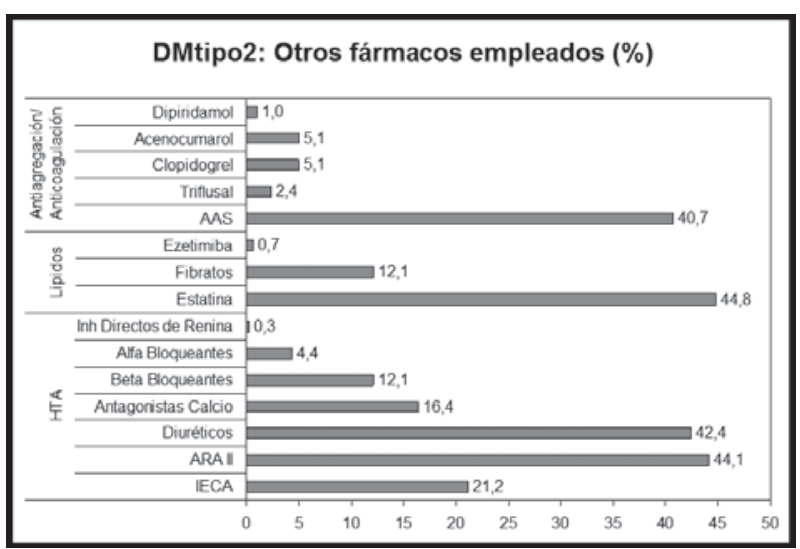

Figura 3. Otros fármacos empleados (\%).

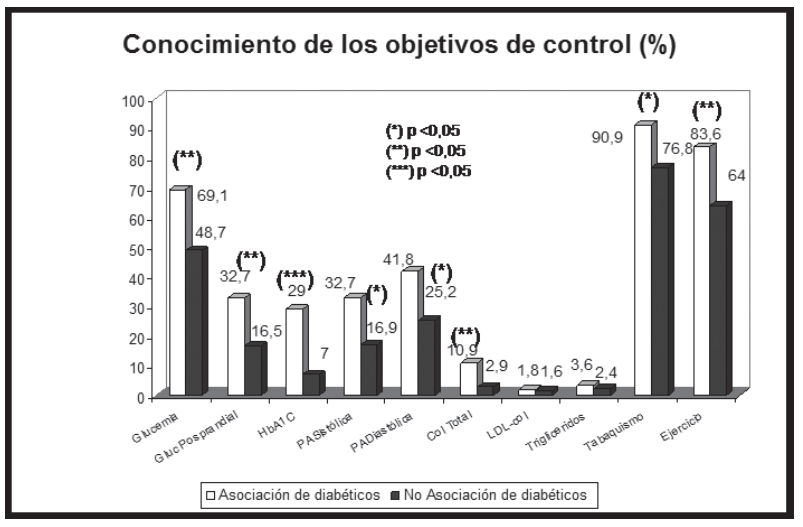

Figura 2. Conocimiento de los objetivos de control según pertenencia o no a asociación de diabéticos.

rior a 6 meses). En concreto: glucemia basal, HbA1C, colesterol total, HDL y LDL colesterol, triglicéridos, tabaquismo, ejercicio aeróbico, creatinina y microalbuminuria. Se registraron las cifras de presión arterial de la última visita. También se recogieron los fármacos que tomaban los pacientes para el tratamiento de la DM, hipertensión arterial, dislipemia, antiagregantes y/o anticoagulantes, y su adecuación a las recomendaciones ADA 2009 ${ }^{9}$. Igualmente se registraron las respuestas sobre los valores que los pacientes consideraron como objetivo de control.

Los parámetros clínicos se determinaron para todos los pacientes en el laboratorio del hos- 


\begin{tabular}{|c|c|c|c|c|c|c|c|}
\hline \multirow[t]{2}{*}{ CONOCIMIENTO } & \multicolumn{7}{|c|}{ VALORES EN CONSULTA } \\
\hline & Glucemia & HbA1C & PAS & PAD & $\begin{array}{c}\text { Colesterol } \\
\text { Total }\end{array}$ & $\begin{array}{c}\text { LDL- } \\
\text { Colesterol }\end{array}$ & Tabaco (\%) \\
\hline \multicolumn{8}{|c|}{ Glucemia ( $\leq 130 \mathrm{mg} / \mathrm{dl})$} \\
\hline - Sí & $140.4(42.8)$ & $7.26(1.18)$ & $134.1(15,5)$ & $76.2(8.7)$ & $196.3(38.3)$ & $120.50(30,4)$ & $17.9 \% *$ \\
\hline - $\quad$ No & $139.1(34,1)$ & $7.19(1.24)$ & $136.0(14.0)$ & $76.0(8.1)$ & $195.9(38.9)$ & $118.6(34.7)$ & $5.8 \%{ }^{*}$ \\
\hline \multicolumn{8}{|l|}{$\operatorname{GPP}(\leq 180 \mathrm{mg} / \mathrm{dl})$} \\
\hline - $\quad$ Sí & $143.5(43.1)$ & $7.56(1.28)^{*}$ & $136.2(15.9)$ & $76.8(8.7)$ & $192.7(34)$ & $114.6(31.0)$ & $12.5 \%$ \\
\hline - $\quad$ No & $138.8(38.1)$ & $7.14(1.17)^{\star}$ & $134.6(14.6)$ & $76.0(8.2)$ & $196.8(39.5)$ & $120.7(32.5)$ & $12.7 \%$ \\
\hline \multicolumn{8}{|l|}{$\mathrm{HbA}_{1} \mathrm{C}(\leq 7 \%)$} \\
\hline - Sí & $139.7(40.6)$ & $7.23(1.15)$ & $134.7(14.8)$ & $76.3(8.6)$ & $194.4(40.9)$ & $119.9(31.3)$ & $24.2 \%$ * \\
\hline - No & $139.8(36.3)$ & $7.24(1.3)$ & $135.3(15.1)$ & $75.8(8.1)$ & $197.0(37.9)$ & $119.0(34.1)$ & $10.8 \% *$ \\
\hline \multicolumn{8}{|l|}{ PAS $(\leq 130 \mathrm{mmHg})$} \\
\hline - $\quad$ Sí & $140.0(35,7)$ & $7.04(0.7)$ & $131.05(16.0)^{*}$ & $75.7(8.2)$ & $196.2(40.6)$ & $123.91(31.3)$ & $11.6 \%$ \\
\hline - No & $139.7(39.9)$ & $7.28(1.3)$ & $136.04(14,4)^{*}$ & $76.2(8.5)$ & $196.13(38.6)$ & $118.46(32.5)$ & $16.6 \%$ \\
\hline \multicolumn{8}{|l|}{$\operatorname{PAD}(\leq 80 \mathrm{mmHg})$} \\
\hline - Sí & $141.0(41.1)$ & $7.24(1.06)$ & $130.5(14,2)^{*}$ & $73.9(9.0)^{*}$ & $194.2(40,6)$ & $122.2(32.6)$ & $14.3 \%$ \\
\hline - $\quad$ No & $139.3(38.2)$ & $7.23(1.2)$ & $136.8(14,2)^{*}$ & $77.08(8.0)^{*}$ & $196.9(37.8)$ & $118.5(32.2)$ & $11.6 \%$ \\
\hline \multicolumn{8}{|c|}{ Colesterol Total $(\leq 185 \mathrm{mg} / \mathrm{dl})$} \\
\hline - $\quad$ Sí & $134.1(43.2)$ & $7.72(1.58)$ & $135.6(15.3)$ & $76.3(7.3)$ & $190.6(41.6)$ & $119.6(37.0)$ & $0.34 \%$ \\
\hline - $\quad$ No & $140.1(38.8)$ & $7.2(1.18)$ & $134.9(14.9)$ & $76.1(8.5)$ & $196.4(38.4)$ & $119.6(32.1)$ & $11.99 \%$ \\
\hline \multicolumn{8}{|c|}{ LDL-Col ( $\leq 100 \mathrm{mg} / \mathrm{dl})$} \\
\hline - $\quad$ Sí & $120.5(3.6)$ & $6.74(0.82)$ & $135,0(9.3)$ & $79.5(3.1)$ & $180.8(44.3)$ & $109.1(35)$ & $0.68 \%$ \\
\hline - No & $140.1(39.2)$ & $7.23(1.21)$ & $135.0(14.9)$ & $76.1(8.4)$ & $195.6(39.7)$ & $119.8(32)$ & $11.64 \%$ \\
\hline \multicolumn{8}{|l|}{ Tabaquismo } \\
\hline - Sí & $140.7(40.3)$ & $7.25(1.2)$ & $135.1(14.9)$ & $76.0(8.5)$ & $195.8(39.2)$ & $119.5(31.4)$ & $11.0 \%$ \\
\hline - $\quad$ No & $134.6(32,3)$ & $7.12(1.2)$ & $134.5(14.8)$ & $76.9(7.4)$ & $194.0(41.7)$ & $119.2(36.2)$ & $1.4 \%$ \\
\hline
\end{tabular}

Tabla 4. Relación entre conocimiento de valores del control metabólico y valores analíticos. Valores: media $( \pm D E)$ para variables cuantitativas, porcentaje para variables cualitativas. GPP: Glucemia posprandial, PAS/D: Presión Arterial Sistólica/Diastólica. * Diferencia estadísticamente significativa $(p<0,05)$.

pital de referencia (Virgen de Altagracia de Manzanares).

Se analizó la relación entre grado de conocimiento de los criterios de control adecuado ${ }^{4}$ y los parámetros analíticos metabólicos.

Se obtuvieron de esta manera 336 HRD (desestimándose 39 por diversos motivos), resultando válidas 297 HRD. Para el estudio descriptivo de los datos utilizamos la proporción para las variables cualitativas y la media (con \pm 1 desviación estándar) para las cuantitativas. Para el estudio analítico empleamos la comparación de medias mediante la $t$ de Student y la Chi-cuadrado para la comparación de porcentajes. Calculamos los intervalos de confianza al 95\% (IC 95\%). El análisis estadístico se efectuó con el programa estadístico G-Stat 2.0.

\section{RESULTADOS}

La prevalencia de Diabetes Mellitus encontrada en nuestra población fue del 8,48\% (IC 95\%: 8,03-8,94) en los mayores de 18 años, siendo del 25,28\% (IC
95\%: 23,8-26,8) en la población de 65 y más años. La tabla 1 refleja las características de la muestra. Encontramos en las diabéticas $(n=159)$ una mayor edad $(71,9 \pm 11,1$ años) respecto a los varones $(66,6 \pm 10,34)(n=138)$, y un porcentaje de pacientes con 65 y más años superior, siendo en ambos casos la diferencia estadísticamente significativa ( $p$ $<0,001)$. En cuanto al nivel de escolaridad, destaca un elevado porcentaje de mujeres en el grupo de "sin estudios" (43,9\%, IC 95\%: 46,1-62,0), teniendo más de la mitad de éstas solo estudios primarios, y en muy bajo porcentaje estudios universitarios (1,9\%, IC 95\%: 0,3-5,4). Solamente el 18,5\% (IC 95\%: 14,3-23,5) son miembros de la asociación de diabéticos (sin diferencias entre sexos), si bien los asociados son más jóvenes que los no asociados $(p=0,006)$.

Destaca una alta prevalencia de FRCV asociados aunque sin diferencias significativas entre sexos, excepto un mayor número de varones fumadores $(22,5 \%)$ frente al $6,3 \%$ de mujeres $(p<0,0001)$. Esta diferencia porcentual se repite en cuanto a la existencia de arteriopatía periférica, y se invierte en la 


\begin{tabular}{|c|c|c|c|c|c|}
\hline \multirow{2}{*}{ CONOCI MI ENTO* } & \multicolumn{5}{|c|}{ NI VEL DE ESCOLARI ZACION } \\
\hline & Sin estudios & Primarios & Medios & Universitarios & Significación \\
\hline \multicolumn{6}{|l|}{ Glucemia ( $\leq 130 \mathrm{mg} / \mathrm{dl}$ ) } \\
\hline - SI (\%) & 41.1 & 67.0 & 73.9 & 60 & $<0,01$ \\
\hline - Respuesta correcta (\%) & 34.9 & 64.9 & 66.6 & 60 & \\
\hline \multicolumn{6}{|l|}{$\operatorname{GPP}(\leq 180 \mathrm{mg} / \mathrm{dl})$} \\
\hline - SI (\%) & 7.1 & 25.8 & 43.5 & 20 & $<0,001$ \\
\hline \multicolumn{5}{|l|}{$\mathrm{HbA}_{1} \mathrm{C}(\leq 7 \%)$} & \\
\hline - SI $(\%)$ & 0 & 15.8 & 17.4 & 40 & $<0,001$ \\
\hline - Respuesta correcta (\%) & & 14.5 & 12.5 & 40 & \\
\hline \multicolumn{6}{|l|}{ PAS $(\leq 130 \mathrm{mmHg})$} \\
\hline - SI (\%) & 48.2 & 69.6 & 82.6 & 80 & 0,01 \\
\hline - Respuesta correcta (\%) & 27.9 & 53.6 & 50 & 40 & \\
\hline \multicolumn{6}{|l|}{$\operatorname{PAD}(\leq 80 \mathrm{mmHg})$} \\
\hline - SI (\%) & 45.8 & 67.4 & 82.6 & 40 & 0,001 \\
\hline \multicolumn{6}{|l|}{ Colesterol Total ( $\leq 185 \mathrm{mg} / \mathrm{dl})$} \\
\hline - SI $(\%)$ & 7.0 & 24.0 & 39.1 & 40 & 0,001 \\
\hline - Respuesta correcta (\%) & 0 & 3.9 & 16.6 & 20 & \\
\hline \multicolumn{6}{|l|}{ LDL Colesterol ( $\leq 100 \mathrm{md} / \mathrm{dl})$} \\
\hline - SI (\%) & 0 & 1.68 & 4.35 & 20 & 0,01 \\
\hline - Respuesta correcta (\%) & & 0.56 & 4.16 & 20 & \\
\hline \multicolumn{6}{|l|}{ Tabaco (No Fumar) } \\
\hline - SI $(\%)$ & 67.9 & 85.9 & 95.6 & 80 & 0,01 \\
\hline \multirow{2}{*}{\multicolumn{6}{|c|}{ Ejercicio aeróbico ( $\geq 150$ min/día) }} \\
\hline & & & & & \\
\hline - SI $(\%)$ & 53.8 & 75.4 & 75.0 & 60 & 0,01 \\
\hline - Respuesta correcta (\%) & 38.3 & 55.8 & 70.8 & 40 & \\
\hline
\end{tabular}

Tabla 5. Relación entre conocimiento de valores del control metabólico y nivel educativo. *Para cada fila corresponde: SI (porcentaje que contestan conocer un valor), Respuesta correcta (porcentaje en cada parámetro) (ADA, 2009).

proporción de afectos por neuropatía, mayor entre las mujeres respecto a los varones $(p=0,029)$.

En los parámetros analíticos de la muestra estudiada (tabla 2) destacan con significación estadística los más elevados niveles de HDL-colesterol en mujeres, mayor perímetro abdominal en hombres, así como un más frecuente y prolongado ejercicio físico en varones. La prevalencia conocida de nefropatía es de un 14\% (IC 95\%:10,4-18,6\%), sin embargo, la función renal calculada por el filtrado glomerular estimado ( $\mathrm{FGe}$ ) mediante la fórmula MDRD ha mostrado diferencias significativas entre sexos, destacando un $42,1 \%$ de enfermedad renal crónica (ERC) en mujeres frente al $28,7 \%$ en varones $(p=0,013)$.

En cuanto al porcentaje de control (ADA 2009) (tabla 3), destaca que glucemia basal, hemoglobina glicada, presión arterial sistólica (PAS) y colesterol total están controlados como máximo en la mitad de los diabéticos. Existe un porcentaje mayor de control de la presión arterial diastólica (PAD), HDL-colesterol y triglicéridos, destacando una ma- yor proporción $(93,7 \%)$ de mujeres no fumadoras $(p<0,0001)$ que de varones, $y$ un muy bajo porcentaje de control del LDL-colesterol. Igualmente se ha valorado el control "acumulativo" de parámetros, iniciando con el porcentaje de control de glucemia basal y añadiendo de modo consecutivo un nuevo parámetro a la suma de los anteriores en el orden siguiente: glucemia basal, hemoglobina glicada, PAS, PAD, LDL-colesterol y tabaquismo (tabla 3 y figura 1), destacando que solo el 9,42\% (IC 95\%: $5,0-15,5)$ de varones y el 5\% (IC 95\%: 2.2-9,6) de mujeres tienen todos los parámetros dentro del rango de control.

Respecto al conocimiento por los pacientes diabéticos de sus objetivos de control, éste queda reflejado en la figura 2, donde se incluye la comparación con el conocimiento de los miembros de asociación de diabéticos. Hallamos que existe un elevado porcentaje de diabéticos que conocen que hay que evitar el tabaquismo y que es aconsejable la realización de ejercicio, mientras que otros objetivos de control son menos conocidos: glucemia basal por un $48 \%$, cifras adecuadas de presión arterial por uno 
de cada cuatro (PA diastólica) y uno de cada seis (PA sistólica); sólo el 16,5\% conocen el objetivo de glucemia postprandial y sólo el $7 \%$ conocen los niveles adecuados de $\mathrm{HbA}_{1} \mathrm{C}$. Las cifras de objetivo de control de parámetros lipídicos son aún menos conocidas.

El análisis de la relación existente entre el nivel de conocimientos y los valores analíticos, según conozcan o no el objetivo, se puede apreciar en la tabla 4, donde quedan reflejados los valores. Encontramos que sólo los pacientes que conocen sus cifras de PAS y PAD tienen dichos valores significativamente más bajos que el resto. Curiosamente, aquellos que aciertan a conocer su objetivo de glucemia postprandial tienen peor control metabólico (HBA1C más elevada), y el porcentaje de fumadores es mayor entre los que conocen el objetivo de glucemia basal y de PAS.

El conocimiento de objetivos de control en aquellos que son miembros de asociación de diabéticos $(n=55)$ es escaso (figura 2), si bien es mayor (con significación estadística) en todos los parámetros respecto a aquellos no asociados. El escaso número de respondedores a los valores de LDL-colesterol y triglicéridos impide establecer conclusiones en cuanto a dichos parámetros.

Dado que nos encontramos en un medio rural/urbano, con una población envejecida y elevado porcentaje de pacientes "sin estudios" o "con estudios primarios" ( $80,9 \%$ en varones y $98 \%$ en mujeres), se ha valorado la relación entre conocimiento de los objetivos del control y el nivel educativo (tabla 5), apreciando que a mayor nivel educativo hay un mayor conocimiento de los objetivos ADA 2009, si bien los pacientes con estudios universitarios (quizá mal estimado por ser muy escasos en nuestra muestra) ofrecen menor grado de conocimientos que aquellos con estudios medios.

La antiagregación es usada en el 45,8\% (40,7\% AAS $+5,1 \%$ clopidogrel), mientras que los inhibidores del SRA-A son utilizados en el $65,3 \%$, siendo Ios ARA II más empleados que los IECA ( $44,1 \%$ vs. $21,2 \%$ ). Otros antihipertensivos, como antagonistas de los canales del calcio y betabloqueantes, son utilizados en menor número de casos (sobre todo en pacientes con cardiopatía isquémica). Destaca el uso de diuréticos en el $42,4 \%$ de los pacientes, generalmente asociados con otros fármacos (IECA, ARA II). El 44,8\% de los pacientes toman estatinas, y fibratos el $12,1 \%$. A pesar de no obtenerse un adecuado control de los parámetros lipídicos, sólo en el $0,7 \%$ de los pacientes de la muestra se emplea la ezetimiba (figura 3 ).

\section{DISCUSIÓN}

La prevalencia encontrada de Diabetes Mellitus ha sido 8,48\% (IC 95\%: 8,03-8,94), ligeramente inferior a la que existe en la provincia de Ciudad Real (conocida $9,1 \%$ y oculta $8,6 \%$ ) y en la comunidad de Castilla-La Mancha (conocida 10\% y oculta 7,3\%) ${ }^{18}$. No cabe duda de que deben existir pacientes no diagnosticados, por lo que se debería realizar una búsqueda activa de pacientes entre aquellos que son considerados como población de riesgo.

Hay una elevada prevalencia de FRCV asociados a la diabetes, similar a la existente en otras comunidades en España ${ }^{4,19,20}$. Comparando nuestros datos con los del Estudio ELIPSE ${ }^{8}$, observamos una mayor prevalencia de obesidad $(45,9 \%$ vs. $57,6 \%)$ y de tabaquismo $(9,65 \%$ vs. $13,8 \%)$. En cuanto al grado de control metabólico, e igualmente referido al estudio ELIPSE ${ }^{8}$, hemos encontrado mejor porcentaje en el control tensional y de LDL-colesterol, manteniendo el porcentaje de control de la $\mathrm{HbA}_{1} \mathrm{C}$. Este control de parámetros metabólicos es superior en cuanto a la $\mathrm{HbA}_{1} \mathrm{C}(50 \%$ varones, $50,9 \%$ mujeres) respecto a otros estudios recientes $\left(35,8 \%{ }^{14}\right.$, $39 \%^{21}, 44,3 \%{ }^{22}$ ), o similar ${ }^{23}$. Hallamos un mayor porcentaje de control de los valores tensionales (TAS $43,9 \%$ y TAD $73,7 \%$ ) en este estudio respecto a otros $8,19,22,23$. Igualmente, hay un mayor porcentaje de control de colesterol total y LDL-colesterol respecto a otros estudios ${ }^{19,22}$. Si bien los valores de HDL-colesterol son más elevados en las mujeres que en los hombres, son éstos los que tienen un mayor porcentaje de control $(69,5 \%)$ respeto a las mujeres $(43,6 \%)(p=0,002)$.

De acuerdo a los criterios ADA 2009, hay un 7,07\% (IC 95\%: 4,4-10,6\%) de pacientes diabéticos con todos los parámetros controlados, superior en hombres $(9,4 \%)$ que en mujeres $(5 \%)$, y también superior $(0 \%)$ a los del estudio ELIPSE ${ }^{8}$ y a otros ${ }^{22}$.

Encontramos un mayor uso de antiagregantes, sobre todo AAS, del $31,35 \%$ (ELIPSE $^{8}$ ) al $40,7 \%$ (sin diferencias entre sexos), también superior al de otros estudios $\left(16,1 \%{ }^{19}, 29 \%^{24}\right.$ ó $\left.36 \%{ }^{22}\right)$. Si se incluyen otros antiagregantes y anticoagulantes, el porcentaje se eleva a $53,3 \%$ (superior al $39,4 \%{ }^{24}$.

En este estudio hay un elevado número de pacientes con edad igual o superior a 65 años $(69,7 \%$, IC $95 \%$ : $64,1-78,8 \%$ ), lo que podría hacer pensar 
que la edad influye en el control, pero en el estudio de Del Cañizo Gómez et al ${ }^{25}$ se concluye que los pacientes diabéticos de edad avanzada de la población estudiada presentan un control metabólico similar a los pacientes más jóvenes, pero con un mejor control de los FRCV asociados.

Respecto al tratamiento farmacológico asociado, solamente dos de cada tres pacientes toman medicación para el bloqueo del SRA-A $(65,3 \%)$ y menos de la mitad $(44,8 \%)$ toman estatinas.

Analizando el grado de conocimiento de los objetivos de control, éste es bajo (sólo el 48,7\% conoce Ios niveles adecuados de glucemia), pero superior a otros estudios donde solo el $35,7 \%{ }^{26}$ y el $42 \%{ }^{13}$ conocían la cifra, mientras que la conveniencia de realización de ejercicio es similar. Destaca el bajo conocimiento de un parámetro tan importante para la prevención de complicaciones como es la $\mathrm{HbA}_{1} \mathrm{C}$ (solamente el $7 \%$ de los pacientes), así como que sólo un 16,5\% conocen las cifras adecuadas de glucemia postprandial, mientras que un elevado porcentaje de pacientes realizan automedida de la glucemia sanguínea mediante el uso de tiras reactivas (46,4\%, IC 95\%: 40,6-52,3), lo que nos indica que la utilidad real de estas mediciones es escasa, pues no se saben interpretar en muchos casos. El grado de conocimiento está directamente relacionado con el nivel educativo, al igual que ocurre con otros padecimientos $\operatorname{crónicos}^{27}$, de modo que a mayor nivel de estudios hay un mejor nivel de conocimientos. Igualmente hemos encontrado una escasa asociación entre el conocimiento de los valores adecuados (ADA 2009) y los valores analíticos, destacando incluso un mayor promedio de $\mathrm{HbA}_{1} \mathrm{C}$ en los que conocen las cifras de glucemia postprandial $(7,56$ $\pm 1,28)$ que en los que no la conocen $(7,14 \pm 1,17)$ $(p=0,0194)$.

En el grupo de pacientes miembros de asociación de diabéticos existe un mayor conocimiento de los objetivos de control, siendo pequeña la diferencia para tabaquismo y realización de ejercicio, máxima para el conocimiento de los valores adecuados de $\mathrm{HbA}_{1} \mathrm{C}(7 \%$ frente a $29 \%)$ e intermedia, pero próxima a veces al $50 \%$, en el resto de parámetros. Los objetivos de LDL-colesterol y de triglicéridos se conocen poco en ambos grupos. No hemos encontrado estudios en la bibliografía que comparen estos grupos, por lo que sólo podemos describir e interpretar nuestros resultados. De todas formas, se podrían considerar como bajos y alejados de lo que consideramos deseable, por lo que podemos concluir que existe un inadecuado control metabólico en la población estudiada, siendo mejor en el grupo de pacientes miembros de asociación de diabéticos. Entendemos aconsejable incidir en la educación diabetológica global en todos los aspectos de la enfermedad y no sólo en los objetivos de control, implicando tanto a médicos como a personal de enfermería (y todo ello coordinado desde el inicio con los otros niveles asistenciales), ya que la mejora del control glucémico está basada en la participación activa del enfermo en la enseñanza-aprendizaje ${ }^{26}$. Tanto la enseñanza individual como la grupal son igual de eficaces ${ }^{28}$, si bien la grupal, como complemento de la educación individual, permite economizar personal y tiempo. La educación diabetológica basada en el modelo PRECEDE es un método útil en el tratamiento integral del paciente con diabetes tipo $2^{29}$. Un reciente estudio ha demostrado que mediante el Programa Diabetes First ${ }^{30}$ una intervención de enfermería (previa formación específica) en colaboración con el médico de familia consigue en sólo tres meses una reducción media de la $\mathrm{HbA}_{1} \mathrm{C}$ del $0,5 \%$, aunque este efecto se diluye a partir del tercer mes.

Finalmente no debemos olvidar luchar contra la inercia terapéutica e implicar al paciente de modo activo en la consecución de los objetivos de control adecuados, no conformándonos con un control "aceptable" y teniendo presente el control integral del diabético, que en ocasiones será casi imposible de conseguir.

En conclusión, el grado de control, de acuerdo a los criterios ADA 2009, de los pacientes diabéticos es bajo e inferior a lo deseable, aunque ha mejorado en los últimos años respecto a otros estudios ${ }^{17}$, habiéndose incrementado el porcentaje de pacientes con los parámetros dentro de objetivo. El grado de conocimiento de los objetivos de control por parte de los pacientes es escaso, siendo mayor, aunque mejorable, en aquellos que son miembros de asociación de diabéticos. El nivel educativo está relacionado directamente con el grado de conocimientos de los objetivos, aunque la escasa representación de los pacientes con formación universitaria nos limita la interpretación en ellos de este parámetro. Mediante la aplicación de la fórmula MDRD se detecta una elevada prevalencia de enfermedad renal crónica "oculta". La implementación de actividades de educación para la salud y la disminución de la inercia terapéutica podrían mejorar a largo plazo el control integral de estos pacientes.

Por último, existen limitaciones para la adecuada interpretación del estudio: 
- Los criterios ADA 2009 de adecuado control metabólico que se han considerado son más estrictos que los previamente existentes, y por ello más difíciles de conseguir en la práctica diaria. Además, el conocimiento de estos criterios por parte de los pacientes es aún más difícil.

- Existe un bajo porcentaje de pertenencia a asociación de diabéticos, lo cual puede dificultar la interpretación del diferente grado de conocimiento de los objetivos de control metabólico.

\section{BIBLIOGRAFÍA}

1. Valdés S, Rojo-Martínez G, Soriguer F. Evolución de la prevalencia de la diabetes tipo 2 en población adulta española. Med Clin (Barc). 2007; 129(9):352-5.

2. Haffner SM, Lehto S, Ronnemaa T, Pyorala K, Laakso M. Mortality from coronary Heart disease in subjects with type 2 diabetes and in nondiabetic subjects with and without prior myocardial infarction. N Eng J Med. 1998; 339:229-4.

3. UKPDS Group. Effect of intensive blood-glucose control with sulphonylureas or insulin compared with conventional treatment and risk of complications in patients with type 2 diabetes (UKPDS 33). Lancet. 1998; 352:837-53.

4. Guerra Bobo A, Cañizo Fernández-Roldán C, Rovira Los$\cos$ A. Prevalencia, grado de control y tratamiento de los factores de riesgo cardiovascular en diabéticos tipo 2 asistidos en un centro de atención primaria. Av Diabetol. 2007; 23(2):131-36.

5. Teixidó M, Prieto G, Ferrer X, Fons A, Monrabá M, Bel C, et al. Grado de control de los factores de riesgo cardiovasculares en pacientes con diabetes mellitus tipo 2. Hipertensión (Madr). 2008; 25(4):151-68.

6. Bernad Vallés M, Velasco Marcos MA, Funcia Barrueco Al, Asensio Calle ML, Rodríguez Borrero R, Maderuelo Fernández JA. Análisis del grado de control integral de los factores del riesgo cardiovascular de los diabéticos tipo 2 . SEMERGEN. 2006; 32(Supl 1):25.

7. Pineda Cuenca M, Custardoy Olavarrieta J, Ortiz Arróniz JM, Cano Montoso JG, Andreu Ruiz MT, Grau C, por el grupo de Investigación Clínica del Sureste (GICS). Grado de conocimiento, tratamiento y control de la hipertensión arterial, hipercolesterolemia y diabetes mellitus en la población general adulta. Aten Primaria. 2004; 33(5):254-60.

8. Galiana Gómez del Pulgar J en representación del Grupo ELIPSE. Efectividad en el control de factores de riesgo cardiovascular en diabéticos tipo 2 de la provincia de Ciudad Real. Rev Clin Esp. 2005; 205(5):218-22.

9. American Diabetes Association. Diabetes Care. 2009; 32(Suppl 1):S13-S61. Disponible en: http://care.diabetesjournals.org/content/32/Supplement 1/S13.Full.pdf/html.

10. Schram TK, Gislasson GH, Kober L, Rasmussen S, Rasmussen JN, Abildstrom SZ et al. Diabetes patients requiring glucosa-lowerin therapy and nondiabetics with a prior myocardial infarction carry the same cardiovascular risk. A Population Study of 3.3 million people. Circulation. 2008; 117:1945-54

11. Aranda P, Tamargo J, Aranda FJ, Luque M, Lopez GarciaFranco A. Use and adverse reactions of antihypertensive drugs in Spain. Part I of the RAAE Study. Blood Pressure. 1997;1(Suppl):11-6.

12. Calderón J, Solís J, Castillo O, Cornejo P, Figueroa V, Paredes $\mathrm{J}$ et al. Efecto de la educación en el control metabó- lico de pacientes con diabetes tipo 2 del Hospital Nacional Arzobispo Loayza. Rev Soc Peru Med Interna. 2003; 16(1):17-25.

13. Gonzalez-Pedraza A, Alvara-Solís EP, Martínez Vazquez R, Ponce-Rosas RE. Nivel de conocimientos sobre su enfermedad en pacientes diabéticos tipo 2 del primer nivel de atención médica. Gac Méd Mex. 2007; 143(6):453-62. Disponible en: www.anmm.org.mx.

14. Bustos Saldaña R, Barajas Martínez A, López Hernández G, Sánchez Novoa E, Palomera Palacios R, Islas García J, et al. Conocimientos sobre diabetes mellitus en pacientes tipo 2 tanto urbanos como rurales del occidente de México. Archivos en Medicina Familiar. 2007; 9(3):147-59.

15. Guzmán-Pérez MI, Cruz-Cauich AJ, Parra-Jiménez J, Manzano-Osorio M. Control glicérico, conocimientos y autocuidado de pacientes diabéticos tipo 2 que asisten a sesiones educativas. Rev Enferm IMSS. 2005; 13(1):9-13.

16. Muñoz Hernández JG, Hernández Pérez MM, Muñoz Hernández H. Controversias sobre educación diabetológica. Salud Rural. 2007; 24(5):29-45.

17. Erdozain Eguaras MA, Unanue Urquijo S, March Nogué J, Peracaula Boschsacoma C, Gelabert Ribas D, Tobias Castella F. ¿Mejora el grado de control metabólico y de conocimientos en diabéticos tipo 2 tras una intervención de educación sanitaria grupal? Aten Primaria. 2000; 26(Supl. 1):355-6.

18. Ballester Herrera MJ, Giralt Muiña P, Gutiérrez G, Sánchez $G$, Angulo JJ, Botella $F$ et al. Estudio epidemiológico de Diabetes en Castilla-La Mancha. Comunicación presentada en el XX Congreso de la Sociedad española de Diabetes. Tenerife, 2009.

19. Jurado J, Ybarra J, Solanas P, Caula J, Gich I, Pou JM et al. Prevalence of cardiovascular disease and risk factors in a type 2 diabetic population of the North Catalonia diabetes study. J Am Acad Nurse Pract. 2009; 21(3):140-8.

20. Benito López P, García Mayor R, Puig Domingo M, Mensa Manteca J, Pallardo Sánchez LF et al. Perfil de los pacientes con diabetes mellitus tipo 2 en la Atención Primaria española. Rev Clin Esp. 2004; 204(1):18-24.

21. Lahoz-Rallo B, Blanco-González M, Casas-Ciria I, MartínAndrade JA, Méndez-Segovia JC, Moratalla-Rodriguez G et al. Cardiovascular disease risk in subjects with type 2 diabetes mellitus in a population in southern Spain. Diabetes Res Clin Pract. 2007; 76(3):436-44.

22. Díaz Grávalos GJ, Palmeiro Fernández G, Casado Górriz I, Arandia García M, Portuburu Izaguirre MM, Vázquez Fernández LA. Cumplimiento de los objetivos de control metabólico en diabetes mellitus en el medio rural en Ourense, Spain. Rev Esp Salud Pública. 2006; 80(1);67-75.

23. Orozco-Beltrán $D$, Gil-Guillen VF, Quince $F$, Navarro-Pérez J, Pineda M, Gómez-de-la Cámara A et al. Collaborative Diabetes Study Investigators. Control of diabetes and cardiovascular risk factors in patients with type 2 diabetes in primary care. The gap between guidelines and reality in Spain. Int J Clin Pract. 2007; 61(6):909-15.

24. Sicras Mainar A, Navarro Artieda R, Rejas Gutiérrez J, Fernández de Bobadilla J, Frías Garrido X, Ruiz Ruiz R. Use of aspirin for primary and secondary prevention of cardiovascular disease in diabetic patients in an ambulatory care setting in Spain. BMC Family Practice. 2007; 8:60.

25. Del Cañizo Gómez FJ, Segura Galindo A, de Gorospe Pérez-Jauregui C, Moreno Ruiz I, González Losada T, Silveira Rodríguez MB. Control de los factores de riesgo cardiovascular en pacientes con diabetes mellitus tipo 2 de acuerdo con su edad. Comunicación presentada en el 52 Congreso Nacional de la Sociedad Española de Endocrinología y Nutrición. Salamanca, 26-28 de mayo de 2010.

26. Zafra JA, Méndez JC, Failde I. Nivel de conocimientos y autocuidados de los pacientes diabéticos atendidos en un 
centro de salud de El Puerto de Santa María (Cádiz). Endocrinología y Nutrición. 2001; 48(7):187-92.

27. Subramanian U, Hofer TP, Klamerus ML, Zikmund-Fisher BJ, Heisler M, Kerr EA. Knowledge of blood pressure targets among patients with diabetes. Prim Care Diabetes. 2007; 1(4):195-8.

28. Dalmau LLorca MR, García Bernal G, Aguilar Martín C, Palau Gaindo A. Educación grupal frente a individual en pacientes diabéticos tipo 2. Aten Primaria. 2003; 32(1):36-41.

29. Salinero-Fort M, Arrieta-Blanco F, Carrillo-de Santa Pau E, Martín-Madrazo C, Piñera-Tames M, Vázquez-Martínez C et al. Eficacia del modelo PRECEDE, de educación para la salud, en el control metabólico y de los factores de riesgo cardiovascular en pacientes con diabetes mellitus tipo 2 . Rev Clin Esp. 2009; 209(7):325-31.

30. Martínez Pastor A, Leal Hernández M, Martínez Navarro A, Navarro Oliver AF, Lifante Pedrola Z, Gómez Ruiz M. Efectos de un programa de revisión en el control de los diabéticos tipo 2 seguidos en atención primaria. Programa Diabetes First. Endocrinol Nutr. 2010; 57(1):16-21 